\title{
Germanica
}

\section{Die Faszination der Form. Robert Musil und die Krisen der Moderne}

La fascination de la forme. Robert Musil et les crises de la modernité

\section{Alice Bolterauer}

\section{(2) OpenEdition}

\section{Journals}

Édition électronique

URL : http://journals.openedition.org/germanica/1802

DOI : 10.4000/germanica. 1802

ISSN : 2107-0784

Éditeur

Université de Lille

\section{Édition imprimée}

Date de publication : 30 juin 2004

Pagination : 19-36

ISBN : 9782913857131

ISSN : 0984-2632

Référence électronique

Alice Bolterauer, «Die Faszination der Form. Robert Musil und die Krisen der Moderne », Germanica

[Online], 34 | 2004, Online erschienen am: 11 Oktober 2012, abgerufen am 06 Oktober 2020. URL :

http://journals.openedition.org/germanica/1802 ; DOI : https://doi.org/10.4000/germanica.1802

Ce document a été généré automatiquement le 6 octobre 2020.

(c) Tous droits réservés 


\title{
Die Faszination der Form. Robert Musil und die Krisen der Moderne
}

\author{
La fascination de la forme. Robert Musil et les crises de la modernité
}

\author{
Alice Bolterauer
}

1.

Robert Musils literarische Selbstreflexion fokussiert von allem Anfang an auf den Aspekt des Nicht-Konsumierbaren, des Nicht-Vereinnahmbaren, des Nicht-eindeutigZuordenbaren, den er als differenzierende Qualität des literarischen Diskurses zur Diskussion stellt. Von seinem ersten literarischen Essay an, der wie selbstverständlich über die Grenzen - moralische wie ästhetische - literarischer Textproduktion reflektiert (« Das Unanständige und Kranke in der Kunst », 1911), bildet das Beharren auf der verstörenden Wirkung moderner Literatur so etwas wie einen Angelpunkt seiner kritischen Auseinandersetzung mit Literatur resp. mit der Frage nach ihrer Berechtigung und ihren sozialen Funktionen in moderner Gesellschaft. Diese Frage, die als nicht-zu-beantwortende oder besser: als immer wieder neu zu beantwortende gedacht werden muss, taucht als Grundsatzfrage in Musils Essays, Tagebüchern und literarischen Texten immer wieder auf. "Aber warum schreibt man denn Kunst? $»^{1}$, lautet ihre einfache Formel. Auf der Suche nach möglichen Antworten, die im Falle Musils über eine «bloß " ästhetische Dimension hinaus durchaus auch existentielle Bedeutung haben ${ }^{2}$, gewinnt die Nachzeichnung der Eigenheit des literarischen Diskurses immer genauere Konturen. Sie wandelt sich von der abwehrenden Distanz all dem gegenüber, was Literatur nicht ist oder nicht sein sollte, zur immer spezifischeren Festlegung dessen, was als grundlegende Kapazität und als entscheidende Differenz des Literarischen angesehen werden kann. In nuce zeigt sich dieses Vorgehen bereits anhand einer kurzen Passage aus einem unvollendet gebliebenen "Profil eines Programms » aus dem Jahr 1912. Was als essentielle Frage nach der Literatur beginnt und sich in einer Reihe von Ausgrenzungen fortsetzt (Kunst ist nicht Rhetorik, ist nicht Wissenschaft, ist nicht diffuse Gefühligkeit), kulminiert letztlich in einer vagen 
Beschwörung der "Art ihrer Verflechtung », d.h. einer formalen Kategorie, die freilich hier noch ziemlich unbestimmt bleibt :

Aber warum schreibt man denn Kunst? Um Dinge noch einmal zu sagen? Es war einmal berechtigt, aber wir sind keine Rhapsoden. Warum beschäftigt man sich nicht mit dem physikalischen Relativitätsprinzip, mit den logisch-mathematischen Paradoxas Couturat's, mit... ?

Weil es Dinge gibt, die sich nicht wissenschaftlich erledigen lassen, die auch nicht mit den Zwitterreizen des Essays zu fangen sind, weil es Schicksal ist, diese Dinge zu lieben, Dichterschicksal. Gefühle $u[n d]$ Gedanken sind unpersönlich $u[n d]$ unkünstlerisch, die Art ihrer Verflechtung ist die Persönlichkeit u. ist die Kunst. (8 : 1317)

Diesen Gedankengang nachzuvollziehen, der aus der Erfahrung einer krisenhaften Moderne die Infragestellung aller herkömmlichen Identitäten und Ganzheiten provoziert, um in der Reflexion auf Schreiben und Literatur doch so etwas wie Identität festmachen zu wollen, unternimmt dieser Beitrag. Er setzt die Krisen der Moderne als unhintergehbaren Background, der die literarische Selbstreflexion in Gang setzt, sie befördert und in dem die literarische Selbstreflexion keinen Halt mehr findet. Dass die Literatur kein Heilmittel angesichts der zahlreichen und durchaus massiven Auflösungserscheinungen der Moderne sein kann, aber dennoch und eben in der Reaktion auf diese ihre Eigenheit zu erweisen hat, macht das Paradoxon Musil'schen Schreibens und seines dieses Schreiben begleitenden Reflektierens aus.

\section{2.}

Es ist die Krise der Identität resp. die Einforderung derselben, die das Schreiben Robert Musils in seinen Intentionen und Ausformungen prägt. "Anspruchsvolles ", d.h. in intellektueller Hinsicht «anspruchsvolles » Schreiben in der Moderne ist für Musil dadurch gekennzeichnet, dass es sich - in Zeiten der Auflösung ganzheitlicher Weltbilder und eindeutiger Identitäten - den diversen Identitätsangeboten widersetzt ${ }^{3}$.

In seiner skeptischen Distanzhaltung gegenüber "falschen», weil voreiligen Identifizierungen gerät Robert Musil nicht nur ein essentialistischer Identitätsbegriff, der die Identität eines Dings, eines Sachverhalts in seinem So-Sein fasst, zum Problem, sondern selbst die Annahme eines funktionalen Identitätsbegriffs, der die Frage nach der Bildung von Identität in den Vordergrund stellt, wird ihm obsolet. Nicht nur verstehe sich von selbst, dass - für ein modernes Bewusstsein in moderner Zeit - nichts "wirklich identisch mit sich selbst ${ }^{4}$ sei, sondern selbst die Prozesse fragiler Identitätsbildung gelte es zu hinterfragen. Wobei Robert Musil weniger die Frage nach dem Wie der Identitätsbildung beschäftigt, sondern ihm vielmehr die Frage nach dem Warum, die fragwürdige Notwendigkeit, überhaupt Identitätsbildungen vorzunehmen, zum Stein des Anstoßes wird. Der Begriff der «Identität » wird bei Musil, bei dem freilich von «Identität» als Terminus nicht die Rede ist, grundsätzlich negativ konnotiert. Die durch vereinfachende, verfälschende Identifikation Form gewordene Identität wird als Inbegriff erstarrter Denk- und Lebensmuster begriffen, denen die intellektuelle Anstrengung der Identitätsverweigerung entgegenzustellen sei, wie Musil es in seinem « Möglichkeitssinn » formuliert :

Wenn man gut durch geöffnete Türen kommen will, muß man die Tatsache achten, daß sie einen festen Rahmen haben : dieser Grundsatz, nach dem der alte Professor immer gelebt hatte, ist einfach eine Forderung des Wirklichkeitssinns. Wenn es 
aber Wirklichkeitssinn gibt, und niemand wird bezweifeln, daß er seine Daseinsberechtigung hat, dann muß es auch etwas geben, das man Möglichkeitssinn nennen kann. $(1: 16)$

5 Die Musil'sche Verweigerung gegenüber dem - um im Jargon der Postmoderne zu sprechen - «Terror der Identitätslogik » evoziert all jene Vorbehalte, Kritikpunkte und Neuansätze, die wir aus dem Diskurs der philosophischen Postmoderne kennen. Sie fordert ein Bewusstsein des «Differenten» und einen Einspruch gegen das " Allgemeine » und gegen "Verallgemeinerungen », jenen " Krieg dem Ganzen », von dem etwa Lyotard spricht und der in einem Plädoyer für das « Nicht-Darstellbare » mündet ${ }^{5}$.

6 Im Folgenden soll weniger von jenen Krisen des modernen Ich die Rede sein, die gleichwohl zum Inventar der Wiener Moderne zählen, vielmehr soll unser Augenmerk jenen Auswirkungen einer generellen Identitätsverweigerung gelten, die sich auf Wahrnehmung, Sprache, Erkenntnis und Erzählen beziehen - auf jene Bereiche mithin, die als Stammbereiche modernen Schreibens dieses bestimmen und erst eigentlich ermöglichen. Was subsumierend als «Sprach-», "Wahrnehmungs-» und «Erkenntniskrise » sowie als « Krise des Erzählens » gefasst werden könnte, meint auch hier, im « Rahmen » des Werks Robert Musils, weniger eine existentielle Erfahrung der Krise als eine Verweigerung gegenüber den auf Identität setzenden, eindeutigen Identifizierungen und Ganzheitskonstruktionen Vorschub leistenden Identitätskonzepten in der Moderne.

\section{3.}

7 Im Jahr 1906 - Jahr des Erscheinens seines Romanerstlings «Die Verwirrungen des Zöglings Törleß», damit aber auch ein Jahr nach der Unentschlossenheit zwischen einer Karriere als Schriftsteller oder aber als Wissenschaftler - entwickelt Robert Musil seinen Farbvariationskreisel, ein Gerät, das bis heute seinen Namen trägt (« Musilscher Farbkreisel») und das als durchaus brauchbar auch im Handel angeboten wurde (zu einem Preis von damals 225 Mark). Dieses Gerät, das an sich als unbedeutend und wenig auffällig erscheint, ist insofern symptomatisch, als es der Wahrnehmung dient und insofern im Kontext zeitgenössischer philosophisch-physiologischer Untersuchungen zu sehen ist, die im Wesentlichen Fragen der Wahrnehmung betreffen. Das, was mit Hilfe dieses Geräts bewirkt werden soll, nämlich die experimentelle Veranschaulichung aller Farbnuancen, die mittels zweier ineinander geschobener Farbblätter erzeugt werden können, zielt auf eine möglichst genaue Analyse des Zustandekommens eines sinnlichen Ausdrucks und dessen Wahrnehmung. Noch im «Mann ohne Eigenschaften » wird Robert Musil die Ungenauigkeit unserer Farbkennzeichnung bemängel ${ }^{6}$ und damit den Fokus auf Wahrnehmungsmuster, Wahrnehmungsgewohnheiten legen, die uns daran hindern, die Dinge so wahrzunehmen wie sie sind.

Der Autor Musil entwickelt nun - ähnlich wie der Techniker Musil - eigene Verfahren, um unsere alltägliche Wahrnehmung zu "verfremden" - die Parallele zu den russischen Formalisten ${ }^{7}$ ist frappant, aber wohl zufällig -, um so den Reflex des allzu Bekannten, d.h. um den Reflex der sofortigen Vereinnahmung unter bereits bekannte Wahrnehmungsmuster und Wahrnehmungskomplexe zu verhindern. 
Eine der auffallendsten Strategien, die Musil einsetzt, um dem formelbildenden Zwang der Wahrnehmungsgewohnheit zu entkommen, ist der Blick durch das Fernrohr, das Triëdere, das Vergrößerungsglas. Das, was sich bei dem Blick durch das Vergrößerungsglas dem wahrnehmenden Bewusstsein darbietet, ist eine fremde Welt, eine nie zuvor gesehene, die nicht länger den Gesetzen und Schemata herkömmlicher Wahrnehmung subsumierbar ist, die inkompatibel, fremd und verstörend wirkt. Als eine zum ersten Mal gesehene, oder besser : als eine zum ersten Mal so gesehene Welt provoziert sie neue Wahrnehmungen, neue Ein- und Ansichten - und zwar sowohl in unserem Wahrnehmungsapparat wie an den Dingen selbst, die als "andere" erscheinen und ein "anderes", geheimes Sein offenbaren, von dem wir in unserem Wahrnehmungsalltag nichts ahnen können: Die Bewegung einer Straßenbahn wird dann zum kubistischen Riesenexperiment - «eine unerklärliche Gewalt drückt plötzlich diesen Kasten zusammen wie eine Pappschachtel, seine Wände stoßen immer schräger aneinander, gleich wird er platt sein» (7: 579) -, der Blick auf ein haushaltstaugliches Fliegenpapier entlarvt dann ein schauriges Kriegsspektakel - « So liegen sie da. Wie gestürzte Aeroplane, die mit einem Flügel in die Luft ragen. Oder wie krepierte Pferde » $(7: 477)$.

10 Das, was als Prinzip der sog. Wahrnehmungskrise der Jahrhundertwende von 1900 angesehen werden kann : das Zerbrechen einer naiven Wahrnehmungsgewissheit, wird von den Autoren der Wiener Moderne - und Robert Musil ist gerade in dieser Hinsicht zu ihren wichtigsten Vertretern zu zählen - sowohl als Motor zur Entwicklung neuer Schreibweisen wie als Impuls zur «Erfindung » der Wirklichkeit angenommen. An sie gekoppelt sind Phänomene des Wirklichkeitsverlusts und der Sprachkrise. Kommunikation erscheint als doppelt falsche Fortsetzung eines falschen Wirklichkeitsbegriffs, der auch die Möglichkeiten der Wirklichkeitserkenntnis in Mitleidenschaft zieht.

\section{4.}

11 Der Infragestellung gängiger Wahrnehmungskonventionalität korreliert eine Erkenntnisskepsis, die sich gleichermaßen auf unsere Erkenntnismodi wie auf die zu erkennenden Gegenstände und Sachverhalte bezieht. Wie können wir - so würde die eine Frage lauten - eine Wirklichkeit adäquat erkennen, wenn wir sie gar nicht " richtig ", d.h. in ihrem objektiven So-Sein, wahrnehmen? Und was - so die zweite Frage - vermögen wir an einer Wirklichkeit $\mathrm{zu}$ erkennen, die sich uns nur fragmentarisch, rudimentär, bruchstückhaft offenbart? Im Gegensatz zu einem radikalen Erkenntnispessimismus unternimmt und inszeniert Robert Musil sowohl sein literarisches wie auch sein essayistisches Schreiben als Medium und Forum «neuer " Erkenntnisse und «neuer » Erkenntnismodalitäten. Musil stellt sein Schreiben unter das Postulat einer ästhetischen Erkenntnis; er fordert von seinem Schreiben Erkenntnis ein - und zwar eine Form von Erkenntnis, die sich jenen, von den exakten Naturwissenschaften, von der Technik und von der Mathematik vernachlässigten Gebieten (wie jenem der Moral, der Gefühle, der Intuition) widmet und die aus der Auseinandersetzung mit diesen auch neue Formen der Erkenntnis entwickelt, zu neuen Erkenntnisformen vorstößt. Ständiger Referenzpunkt dieses Unterfangens bleibt die Erkenntnisfähigkeit der Naturwissenschaften, die zum einen - in ihrer Stringenz, in 
ihrer « Kühnheit $»^{8}$ - weiterhin als Vorbild und Ansporn fungiert und die zum anderen - in ihrer Einseitigkeit und Formelhaftigkeit - überboten werden soll.

Was Robert Musil in seinen Aufsätzen und Essays immer wieder akribisch genau anführt, dem er sich annähert, das er nuanciert und variiert und weiterschreibt und umschreibt : eine Beschreibung und Rechtfertigung dessen, was ein in intellektueller Hinsicht anspruchsvolles Schreiben sein könnte/sollte, versucht er in seinen fiktionalen Texten « umzusetzen », d.h. seine eigene Literatur als mögliche Antwort zu entwerfen angesichts des « Ungenügens », das ein Großteil vorhandener Literatur ihm verursacht.

13 Während in seinem frühen Essay "Der mathematische Mensch» (1913) noch der Mathematiker als der alleinige (oder zumindest als der allein relevante) Prototyp des Intellektuellen verstanden wird und seine Methoden, seine Verfahren als Vorbild auch für die literarische Auseinandersetzung mit dem Nicht-Rationalen angesehen werden, geht Robert Musil in seinem fünf Jahre später entstandenen Essay «Skizze der Erkenntnis des Dichters " (1918) einen Schritt weiter und behauptet, wie der Titel des Essays bereits signalisiert, eine Eigenständigkeit dichterischer Erkenntnis. In diesem Aufsatz wird die Möglichkeit einer dichterischen Erkenntnis erwogen - und zwar als eine der rationalen Form der Erkenntnis gleichberechtigte Form der Erkenntnis, die sich zwar weiterhin in ihren Intellektualitätsstandards an der Mathematik und den Naturwissenschaften orientiert (d.h. die klar, genau, logisch verfährt), aber aufgrund ihres anderen Gegenstandes doch "anders » ist, anderen Gesetzen gehorcht und anderen Notwendigkeiten.

Musil unterscheidet hier den rationalen Menschen auf ratioïdem Gebiet und den rationalen Menschen auf nicht-ratioïdem Gebiet. Für das erste stehe der Mathematiker, für das zweite der Schriftsteller. Das « ratioïde » Gebiet umfasse - sagt Musil - «alles wissenschaftlich Systematisierbare, in Gesetze und Regeln zusammenfaßbare [!] », es sei gekennzeichnet «durch eine gewisse Monotonie der Tatsachen, durch das Vorwiegen der Wiederholung, durch eine relative Unabhängigkeit der Tatsachen voneinander ", vor allem aber dadurch, "daß sich die Tatsachen auf diesem Gebiet eindeutig beschreiben und vermitteln lassen ». ( $8: 1026 f)$ All das gelte für das «nichtratioïde" Gebiet nicht. Sei das ratioïde Gebiet « das der Herrschaft der ,Regel mit Ausnahmen'«, so sei das nicht-ratioïde Gebiet das «der Herrschaft der Ausnahmen über die Regel " $(8:$ 1028). Was nur ein "gradueller Unterschied» scheine, sei tatsächlich von so fundamentaler Bedeutung, dass er « eine vollkommene Umkehrung der Einstellung des Erkennenden » verlange : «Die Tatsachen unterwerfen sich nicht auf diesem Gebiet, die Gesetze sind Siebe, die Geschehnisse wiederholen sich nicht, sondern sind unbeschränkt variabel und individuell » $(8: 1028)$.

Die Beschäftigung mit dem Bereich des Nicht-Ratioïden, die zu den Aufgaben der Literatur zähle, beansprucht aus der Sicht Musils die Entwicklung eigener Erkenntnisformen, dichterischer Erkenntnisformen, die auf die völlig andere Qualität nicht-ratioïder Gegenstandsbereiche Rücksicht nehmen, aber deswegen nicht weniger anspruchsvoll sein dürfen.

Die ästhetischen Experimente, die Musil in seinen in einem engeren Sinne « literarischen » Texten vollführt, lassen sich als Konsequenz der von ihm behaupteten und geforderten dichterischen Erkenntnisqualität begreifen, d.h. sie sind gleichermaßen als Ausdruck eines Erkenntniswillens zu sehen wie als experimentelle Formen literarisch-ästhetischer Erkenntnis. Sie setzen den epistemologischen 
Anspruch voraus und verstehen sich zugleich selbst schon als experimentelle Varianten eines erst $\mathrm{zu}$ erreichenden literarischen Erkenntnisraums. Was in den einzelnen Texten als konstanter Faktor auftritt, ist ein seltsames Ineinander von diskursiver Hereinholung der Reflexion auf eine erst zu erahnende Form dichterischer Erkenntnis, von der gesprochen wird und der man sich sprechend anzunähern versucht - ",Dinge sind es, 'meinte er, ,hinter dem Horizont des Bewußtseins' » (6: 196) -, und einer damit zusammenhängenden Auflösung kohärenter Handlung in ein Bewusstseinskontinuum, das dieses Annähern bereits ist.

Da in diesen Texten ein epistemologischer Anspruch im Vordergrund steht und nicht ein narrativer oder besser : der epistemologische Ansatz den narrativen in sich birgt, wird das Erzählen zugunsten des Erkennens zurückgestellt. Musils Ungeschick im Erfinden von Fabeln, das von der Forschung wiederholt konstatiert worden ist, findet sich hier aufgehoben in dem völlig anderen Anspruch, mit dem er an sein Schreiben tritt. Nicht die in narrativer Hinsicht prächtige Ausgestaltung der Fabel steht hier im Vordergrund, sondern ihre möglichst präzise, genaue, detailreiche Erkenntnis. Die Handlung wird zerlegt wie ein philosophisches Theorem, sie wird geprüft wie ein theoretisches Argument und sie wird experimentell überprüft wie eine physikalische Versuchsanordnung. Das Ende könnte sein : nicht der definitive Beschluss, nicht das neue Gesetz, sondern die Verfeinerung der Analysemethoden. Da Musil auf dem Gebiet der angewandten ästhetisch-literarischen Erkenntnis Neuland betritt und dieses per se das Nicht-Systematisierbare, das nicht in Gesetzen zu Fassende darstellt, sind auch die auf dem Gebiet der Literatur anzustrebenden Erkenntnisse immer nur solche der Intuition und der Vorläufigkeit, nicht aber der subjektiven Willkür. Was Musil in seinem Essay «Skizze der Erkenntnis des Dichters » als Programm formuliert, bleibt auch im Hinblick auf die Radikalität des Anspruchs - gelten : « den inneren Menschen erfinden.» (8: 1029) Wie faszinierend dieser Anspruch auf ein epistemologisch motiviertes und ausgerichtetes Schreiben sein kann, haben im späten 20. Jahrhundert neben anderen Autor/innen insbesondere Ingeborg Bachmann und Dieter Kühn vor Augen geführt.

\section{5.}

18 Ähnlich wie im Falle der Erkenntnisskepsis, wo sich bei Robert Musil weniger eine Erkenntniskrise, d.h. ein Verzweifeln an den Erkenntnisfähigkeiten des Menschen feststellen lässt als vielmehr ein Bemühen um «neue » Formen des Erkennens, verhält es sich bei Musil mit der sog. Sprachkrise. Von einer Sprachkrise im existentiellen Sinn, wie wir sie von Hugo von Hofmannsthals Chandos-Brief kennen, kann bei Musil nicht die Rede sein. Wenn Hofmannsthals Lord Chandos sein Verstummen als Resultat eines Verlusts der Sprache und des Sprechens beschreibt - dass ihm die Worte im Munde zerfielen wie "modrige Pilze ", lautet die berühmte, vielzitierte Phrase -, so wird man ein literarisches Äquivalent dieser Schreib- und Sprachkrise bei Musil vergeblich suchen. Der Einfluss der Nietzsche- und Maeterlinck-Lektüren, der sich auch bei Musil in diversen Anlehnungen, Tagebuchnotizen, Motti ${ }^{10}$ feststellen lässt, evoziert selbstverständlich auch bei ihm eine große Vorsicht gegenüber dem Verfälschungsund Manipulationscharakter von Sprache - auch diese in zahlreichen Nebensätzen, Einschüben, Nebenbemerkungen nachweisbar -, initiiert aber doch vor allem eine Suche nach «neuen» sprachlichen Ausdrucksmöglichkeiten. Der kritischen 
Auseinandersetzung mit der "alten» Sprache unseres Alltags, aber auch der literarischen Tradition korreliert die Arbeit an der «neuen " Sprache. Das, was bei Hofmannsthal als Versagen der Sprache und des Sprechens " an sich» figuriert, wird bei Musil als Versagen einer bestimmten, verbrauchten und missbrauchten Sprache und Sprachverwendung apostrophiert, dem eine gleichermaßen radikale wie unverbrauchte Spracharbeit entgegengesetzt wird.

Natürlich wird auch der junge Törleß von der Angst, « sich nicht verständlich machen zu können» (6:133), geplagt, natürlich weiß auch Musil, dass «alle Worte soviel Nebensinn, Doppelsinn, Nebenempfindung, Doppelempfindung [haben], daß man gut thut sich von ihnen fern $\mathrm{zu}$ halten $»^{11}$, natürlich wird auch ihm das Kommunikationshandicap von Sprache zum Thema - «Aber wie er es aussprach, war es ein entwerteter Begriff und sagte nichts von dem, was er meinte» $(6: 195)$. Dennoch oder gerade deswegen erwächst ihm aus dem Wissen um den defizitären Charakter von Sprache und sprachlicher Äußerung eine radikale Kritik an herkömmlicher Alltagssprache und ein ebenso radikales Bemühen um eine neue Sprache.

Bitterer Spott gilt den abgenutzten, längst verbrauchten, nur noch als Kitsch tauglichen Sprach- und Worthülsen, aus denen sich Alltagskonversation und Literatursprache zusammensetzen. Gerade in der Posse "Vinzenz und die Freundin bedeutender Männer» werden Phrasenhaftigkeit und Sprechen in wohlgeformten Sprachpaketen aufs Korn genommen :

Bärli : Ich entführe Sie, ich reiße Sie an mich!

Alpha: Und dann? Sie können mich doch nicht zeitlebens entführen und unausgesetzt an sich reißen : was wird also dann sein ? Pause

Bärli etwas kleinlaut : Wir werden namenlos glücklich sein.

Alpha : Namenlos?

Bärli : Sicher ! Wir werden namenlos glücklich sein.

Alpha : Sie haben sich das etwas ungenau ausgedacht : Es fehlen Ihnen schon wieder die Namen.

Bärli : Ja, Alpha : mir fehlen die Namen. Mir haben immer die Namen gefehlt, wenn ich etwas gewollt habe. Darum nehme ich es mir! Darum rede ich nicht, wie die andern, sondern nehme es mir! Ich werde Sie auf Händen tragen. Ich werde alle Steine aus Ihrem Weg räumen. Ich werde Sie anbeten. Wir werden uns lieben. (6 : 412)

Was zum Anlass der Posse wird : ein unüberlegtes Reden und Sprechen, das aus bereits fertigen Wortpaketen und Wortzusammenfügungen besteht, die ebenso wohlvertraut klingen wie sie de facto nichtssagend sind, taucht natürlich auch in anderen Diskussions- und Reflexionszusammenhängen auf. Sorgloses Sich-Anvertrauen den Topoi bestimmter Weltanschauungen wird von Musil als bequeme Lüge oder als verlogene Bequemlichkeit entlarvt. All diesen Sprechweisen, sie mögen politisch korrekt sein oder nicht, sie mögen gut gemeint sein oder nicht, haftet als Grundübel die Sorglosigkeit ihrer unbedachten Inanspruchnahme von Silben, Worten, Sprachpartikeln als Requisiten eines Sprechens an, das mehr der Konventionalität ihrer gewohnten Zusammensetzung vertraut als dass es diese wirklich beim Wort nimmt. Die Worte als Worte zu nehmen, sie in der polaren Gerichtetheit ihrer doppelten Verfasstheit aus Bezeichnendem und Bezeichnetem auf ein Gemeintes und ein als Gemeintes zu Verstehendes hin genau zu setzen, darin werden Impuls und Anspruch von Musils Schreiben liegen. Die Worte aus ihren fixen Wortverbindungen zu lösen « Das Wort ist nicht gar so sehr Träger eines Begriffs [...], sondern es ist, wenn es nicht definitorisch zu einem Fachwort eingeengt wird, bloß das Siegel auf einem lockeren 
Pack von Vorstellungen» $(8: 1212 \mathrm{f})$, heißt es -, d.h. den an ein Wort gebundenen « Pack von Vorstellungen » aufzudröseln, auseinanderzunehmen und sie, die Worte, «neu », d.h. auf eine andere, noch unverbrauchte, reflektierte Weise zusammenzustellen, darin besteht das Musil'sche Schreibunternehmen. In gewisser Weise lassen sich auch in Musils Auseinandersetzung mit den Dilemmata und Aporien modernen Schreibens ein gleiches Vorgehen und ein vergleichbares Anliegen wie in seiner Auseinandersetzung mit der Wahrnehmung und unserer Wahrnehmungskonventionalität feststellen. Kernpunkte seiner Reflexion wie seines Schreibens (und seines reflektierenden Schreibens) sind weniger der Zweifel an den Möglichkeiten des Wahrnehmens oder des Sprechens und Schreibens "an sich" als vielmehr die Kritik an den eingefahrenen Mustern des Wahrnehmens und Sprechens resp. Schreibens und das Bemühen, in einem Prozess der Destruktion, des analysierenden und sezierenden Auseinandernehmens, Auseinanderziehens die «alte " Form der Weltaneignung zu zerstören und langsam, in kleinen Schritten der Analyse und Vivisektion zu neuen Einsichten und zu neuen Formen des Wahrnehmens, Sprechens und Schreibens zu gelangen.

22 Am Ende steht das «neue » Sprachuniversum Musil'scher Prägung, von dem er selbst sagt, man müsste es unter Glasplatten legen und Tag für Tag, Seite für Seite auf sich wirken lassen ${ }^{12}$. Es ist ein Sprachraum, in dem die Worte so gesetzt sind, als würden sie eben erst entstanden sein oder aber als würden sie zum ersten Mal zum Einsatz kommen, ihre Verwendung finden als neues Material der Auseinandersetzung mit Welt und Wirklichkeit und als deren Kommunikation. Sie sind Teil eines Universums, das durch sie hergestellt wird und in dem herkömmliche Kategorien des Sprechens und Schreibens wie Chronologie («Es gibt im Leben eine Zeit, wo es sich auffallend verlangsamt... » (6:234)), Topographie (« Dort, in Kakanien... » (1:32)) oder Kausalität («Woraus bemerkenswerter Weise nichts hervorgeht» (1:9)) außer Kraft gesetzt sind. Was sich auftut, ist eine neue Perspektive auf die Welt, die sich der Sprache bedient, die sich erst mit Hilfe von Sprache eröffnet und in der Sprache mehr ist als bloßes SprachMaterial, nämlich: Mittel der Verständigung über eine differenzierter und subtiler wahrgenommene und beschriebene Welt.

6.

Dieser Anspruch - hier in verschiedenen Anläufen immer wieder umkreist und angesprochen - auf ein Durchbrechen stabiler Formen, Muster oder Identitäten (des Wahrnehmens, des Erkennens, des Schreibens und Sprechens) provoziert auch eine kritische Haltung gegenüber herkömmlichen, traditionellen Formen des Erzählens, die entweder eine als sicher geltende Erzähltradition oder aber so etwas wie ein institutionalisiertes mimetisches Urvertrauen weiterschreiben, d.h. die entweder die Sicherheit der narrativen Gattung (dass erzählt werden kann) oder aber die der literarischen Narrativik zugrundeliegende Fähigkeit der Wirklichkeitsabbildung (was erzählt werden kann) kontinuieren. Beides wird dem großen Erzähler der Moderne, Robert Musil, gleichermaßen suspekt. Weder mag er sich der beruhigenden Gewissheit literarischer Tradition (immer ist doch erzählt worden) noch der Vergewisserung literarischen Wirklichkeitsbezugs anvertrauen. Sein Misstrauen gilt ebenso den überlieferten Formen des Erzählens und Darstellens (Novelle, Roman, Drama) wie dem Prozess des Erzählens/Darstellens an sich. Er diagnostiziert im Rahmen des 
zeitgenössischen Schreibens - es mag avantgardistisch, reaktionär, anerkannt oder verboten sein - eine Unbekümmertheit im Umgang mit den Formen des Erzählens und dem Erzählen an sich, die er nicht länger prolongieren möchte. Also nicht die 1000. Novelle nach $999 \mathrm{ihr}$ zuvorgegangenen ähnlichen und nicht eine Naivität des Erzählens, wo das Erzählen selbst an seine Grenze gestoßen ist - «Nicht Erzählung fingieren » (Tgb 1 : 597), heißt es im Tagebuch.

Robert Musils «Krise des Erzählens» ist eine Krise des herkömmlichen, meist realistischen Erzählens und antiquierter Erzählformen. Dem resp. diesen setzt er das Bemühen um eine Erneuerung konventioneller Gattungen und die essayistischtheoretische Hereinholung differenzierter Reflexion über das Erzählen entgegen. Hier ist zum einen auf den - im postmodernen Jargon - «dekonstruktiven » Umgang mit « klassischen» Gattungen der Literatur wie Novelle, Roman und Drama hinzuweisen. Musil verwehrt sich den Gattungserwartungen, er zerstört sie und versucht, durch den Bruch der Erwartungshaltungen zu neuen Formen des Erzählens und Darstellens zu kommen, die weniger abgeschmackt, intellektuell anspruchsvoller sind und vor allem den modernen Lebenswelten besser entsprechen, authentischer sind.

Was Robert Musil bereits in seinen Varianten moderner Novellen vor Augen führt : die Aufhebung klar zu identifizierender Raum- und Zeitgefüge, die Sprengung gewöhnlicher Kausalzusammenhänge, den Verzicht auf Spannungsbögen, wird er in seinem großen Roman « Der Mann ohne Eigenschaften » fortführen. Indem er ironisch alle Konstitutiva der großen Roman-Tradition unterläuft, d.h. den Einsatz von Ort, Zeit, Handlung und Figur $^{13}$ als willkürliches Spiel inszeniert und wieder torpediert, provoziert er eine dynamische Auflösung bestimmter Erzählschemata in einen dicken Brei ihrer Bestandteile, aus denen sie, die Erzählungen, Novellen, Romane, neu zusammengesetzt werden müssen. Besonders im Drama wirkt sich diese andere Akzentuierung in revolutionärer Weise aus. Die Vernachlässigung des dramatischen Konflikts, aus dem alle Handlung entsteht, der Verzicht auf Spannungsentwicklung, auf Spannungsaufbau, Höhepunkt, Retardierung oder Umschwung sowie die Zurücknahme der Helden und ihre Transformation zu monologisierenden Denkern bewirken einen radikalen Bruch mit der Dramentradition, d.h. mit den Erwartungen des Theaterpublikums ebenso wie mit den an Dramenarbeit geknüpften Intentionen und Ansprüchen. Musils Drama - vorbildlich hier vor allem das Stück « Die Schwärmer » will nicht unterhalten, will nicht belehren oder erschüttern, sondern Gewissheiten zerstören - auch die der eigenen Form.

Der andere, zweite Aspekt, der für Musils Anstrengung für eine Revolutionierung herkömmlichen Erzählens wichtig ist, richtet sich auf die Reflexion über das Erzählen selbst. Die Frage, wie Erzählen überhaupt noch möglich sein soll, wenn weder auf Wahrnehmung noch auf Wirklichkeitserkenntnis und Sprache Verlass ist, provoziert eine massive Infragestellung menschlicher Erzählvermögen überhaupt bzw. insbesondere eine Absage an "naive», an Alltagskonversation erinnernde Erzählhaltungen. Die Verzichterklärungen Musils gegenüber dem «naiven », herkömmlichen Erzählen sind Legion. Da ist in seinen Tagebüchern von seiner "Abneigung gegen das Erzählen [...], gegen die Scheinkausalität u[nd] Scheinpsychologie $»^{14}$ die Rede, da erklärt er in Vorentwürfen zu seinem großen Roman, dass die "Geschichte des Romans » darauf hinauslaufe, "daß die Geschichte, die in ihm erzählt werden sollte, nicht erzählt wird » $(5: 1937)$. In diesen Aussagen steht also nicht bloß eine bestimmte, verbrauchte Erzählform zur Disposition, sondern 
die Möglichkeit des Erzählens selbst, des Fingierens von Wirklichkeit wird hier zum Problem. Musil äußert sich skeptisch gegenüber einem mit dem Gestus der Selbstverständlichkeit auftretenden Erzählhabitus, dem wohl das Was seines Erzählens - beispielsweise : wie modern muss modernes Erzählen in seinen Inhalten sein ? - zur Herausforderung wird, dem aber das Wie des Erzählens keinen Gedanken abnötigt. Dementgegen insistiert Musil auf einer reflexiven Betrachtung der Voraussetzungen modernen Erzählens überhaupt; er fragt nach den Bedingungen des Erzählens in der Moderne, nach den Grenzen und Möglichkeiten eines solchen Erzählens und plädiert dafür, diese Reflexion nicht auszusparen, sondern sie selbst in den Erzählvorgang zu integrieren ${ }^{15}$.

Dadurch wird letztlich das Erzählen nicht aufgehoben, es wird nicht das Ende des Erzählens postuliert, aber eine andere Form des Erzählens propagiert, die Erzählen und Reflexion des Erzählens zusammenspannt zu einem «neuen» Erzählen, das die philosophischen, ästhetischen, wahrnehmungsphysiologischen und technischen Komponenten seines Zustandekommens nicht verschweigt, sondern diese $\mathrm{zu}$ integrativen Bestandteilen des Erzählvorgangs nobilitiert. Dann wird es möglich, ein ganzes Romankapitel der "Utopie des Essayismus» zu widmen ${ }^{16}$, d.h. den eigentümlichen, zugleich erzählenden und reflektierenden, eben " essayistischen » Charakterzug von Musils Erzählen selbst zum Thema zu machen, vom je konkreten Erzählen zu abstrahieren und doch gerade diese Abstraktion wieder in die Erzählung zu integrieren.

Was Robert Musil selbst immer wieder als « Schwäche » seines Erzählens kritisiert hat : dieses mangelnde Vertrauen in das eigentliche, «naive » Erzählen, produziert de facto ein höchst anspruchsvolles und reflexiv-theoretisches Erzählgewebe, das zeigt, wie problematisch das Erzählen in der Moderne geworden ist und wie es doch noch funktionieren könnte.

\section{7.}

29 Wodurch aber - so wäre letztlich noch zu fragen - unterscheidet sich ein literarischer Text von einem nicht-literarischen ? Ist diese Frage überhaupt zu stellen ? Oder : wie ist sie zu stellen, will man vorschnelle Identifizierungen - hier Literatur, da NichtLiteratur - vermeiden? Warum kann diese Frage überhaupt wichtig sein/werden, wenn doch im Grunde alles Schreiben nur der Aufdröselung und Auflösung stabil gehaltener und für « wirklich » genommener Identitäten dienlich war? Muss sie sich nicht selbst desavouieren und destruieren angesichts des großen, analytischen und sezierenden Schreibunternehmens Robert Musils? Die "Fakten" sprechen dagegen. Das lebenslange Ringen Robert Musils um ein modernes, zeitgerechtes und anspruchsvolles literarisches Schreiben, das sich in seinen Essays, Aufsätzen, Glossen ebenso niederschlägt wie in seinen in einem engeren Wortsinn "literarischen » Werken, inkludiert immer auch eine Reflexion über Bedingungen, Intentionen und Ziele moderner Literatur. Und auch wenn es als grundlegendes Anliegen modernen Schreibens gelten kann, Identitäten zu zerstören, so erwächst doch dem literarischen Text selbst aus diesem Beharren auf Identitätsverweigerung eine eigene Identität ${ }^{17}$.

Was sich in den diversen Essays anhand von Einzelfragen herauskristallisiert : eine vorsichtige Annäherung an spezifische Problemkomplexe modernen literarischen Schreibens, erfährt in Musils letztem großen literarischen Essay, der nicht zufällig den 
signifikanten Titel « Literat und Literatur. Randbemerkungen dazu » (1931) trägt, seine kritische - auch selbstkritische - Apotheose. Es ist eine große Bilanz zeitgenössischen Schreibens, auch des eigenen Schreibens, und ein Versuch, durch die Vermittlung neuester wissenschaftlicher Erkenntnisse zu einer akzeptablen, wenngleich immer nur vorläufigen Definition modernen literarischen Schreibens zu gelangen.

Die Intention dieses letzten großen Essays ist sowohl eine Rehabilitierung von Literatur in der modernen Gesellschaft wie auch eine ästhetische Festlegung. Er richtet sich gegen die Diffamierung und Marginalisierung des Schriftstellers als Literaten, der zum einen als moralisch unzuverlässig gilt : « er ist Nur-Literat, und daß sich daraus eine geringschätzige Bezeichnung entwickeln konnte, die nicht allzufern den Begriffen Kaffeehaus und Boheme liegt $[. .] ».(8: 1203)$ und der zum andern als nicht vollwertig angesehen wird : « er sei ein Mann, der sich irgendwie zu ausschließlich und auf Kosten seiner, vollen Menschlichkeit' oder mit der Literatur beschäftige " (8: 1204). Dementgegen plädiert Musil für ein «neues » Selbstverständnis des «Literaten », der die Emphase des alten «Dichter »-Begriffs von sich gewiesen hat und gleichwohl selbstbewusst sich zu seiner Zuständigkeit für die Belange der Literatur bekennt : « Wir alle sind zuerst und zuoberst Literaten. Denn Literat im richtigen Sinn, das ist der noch nicht spezifizierte Funktionär der Literatur, das Grundgebilde, woraus alle anderen entstehen.» $(8: 1204)$

Wodurch aber der «Literat» der modernen Gesellschaft sich auszeichne, was seine Literatur kennzeichne, dem versucht Musil vor allem mittels Differenzierung ex negativo näherzukommen. Was den «Literaten» - zur Zeit des Entstehens des Essays ein Wort mit abschätzigem Beigeschmack - von diversen Schreiberlingen zum einen, vom « wahren » Dichter zum anderen unterscheide, ist eben das, wozu die zunehmende Ausdifferenzierung der modernen Gesellschaft - sensu Luhmann ${ }^{18}$ - verpflichtet : die Einführung einer Ebene der Beobachtung zweiter Ordnung. Der «Literat» sei ein "Mensch aus zweiter Hand», so heißt es bei Musil, « der nicht (wie es angeblich der Dichter tut) von den Tatsachen des Lebens abhänge, sondern von den Berichten über sie» (8: 1204). Der «Literat» ist zwar einer, der sich ganz und ausschließlich zur Literatur bekennt, da er ja diese sogar in seinem Namen trägt und sich nicht wie jene, die aus der Literatur ein Geschäft machen, eine "schöne Berufsbezeichnung " zulegt : " sei es auch nur, daß er Zwischentiteldichter heißt » $(8: 1203)$; er ist aber auch einer, dem die Naivität des In-der-Welt-Seins und In-der-Welt-schreiben-Könnens abhanden gekommen ist, dem das Welt-Erleben und das Welt-Beschreiben nicht mehr ineinander übergehen, dem das Letztere nur noch möglich ist, wenn das Erstere durch den Filter der distanzierenden Reflexion hindurchgegangen ist. Die unverzichtbare Reflexionsnotwendigkeit moderner Literatur - der « Literat » sei ein Mensch, « dessen Intellekt mit seinen Gefühlen spielt oder dessen Gefühle mit seinem Intellekt spielen» (8: 1206) - mache das Spezifikum modernen literarischen Schreibens aus; aus ihr resultieren Essayismus, Intellektualismus, Fragmenthaftigkeit und Auflösung der in der Literatur dargestellten Wirklichkeit, d.h. jene von den Lesern und Leserinnen als Defizite empfundenen Phänomene moderner Literatur, die ein «Bedürfnis nach Entschädigung" aufkommen lassen, eine Sehnsucht nach "Originalität, Erlebnis, Reportage und Erhabenheit " $(8: 1207)$, die sich de facto nicht mehr oder nur um den Preis eines nostalgischen Rückschritts befriedigen lasse. Die Literatur der Moderne müsse ihrer Reflexionsnotwendigkeit und ihren Intellektualitätsstandards gerecht werden, d.h. sie müsse mit den " alten» Vorstellungen, was Literatur sei, brechen, eine « neue » Form literarischen Schreibens erst finden und diese installieren. So jedenfalls 
ist wohl der prekäre Satz Musils - «Literatur als Reaktion darauf, daß es keine Literatur gibt » $(8: 1211)$ - zu verstehen : als Absage an eine « alte », fixe, feststehende Vorstellung von Literatur und als Aufforderung zur Neudefinition dessen, was Literatur sein könnte - in dem Wissen, dass es einen allgemeinen Begriff von Literatur ( daß es keine Literatur gibt ») nicht geben kann oder nicht geben muss. Warum also Literatur? Aus einem ästhetischen, wohl aber mehr noch aus einem existentiellen Grund ${ }^{19}$ verbeißt sich Robert Musil in die Frage nach dem Warum der Literatur, die er als Frage nach dem Was und Wie der Literatur apostrophiert, d.h. als Frage nach der Funktion von Literatur und nach ihrem Code fokussiert. Mag die Frage nach der Funktion modernen literarischen Schreibens für Musil unbestritten in der Beeinspruchung falscher Identitäten und Ganzheiten ihre Antwort finden, so betritt er mit der Frage nach dem Wie das schwankende Neuland ästhetischer Spekulation. Was unterscheidet einen literarischen Text von einem nicht-literarischen Text? Zur Beantwortung dieser Frage bemüht Musil nun - einigermaßen überraschend - den alten Begriff der " Form », mit dem er zugleich an die traditionelle Dichotomie von «Inhalt » und «Form » anschließt und diese zugleich revolutioniert. Indem er nämlich an neueste Erkenntnisse der von Christian von Ehrenfels begründeten Gestaltpsychologie anknüpft, deren Weiterführungen er in Berlin kennengelernt hat ${ }^{20}$, versucht er, den Begriff der «Form» weiter zu nutzen und diesem zugleich einen neuen Inhalt $\mathrm{zu}$ geben. Im Unterschied zur herkömmlichen Auffassung von «Form » als geordnetem Zusammenhang von Einzelteilen besteht Musil - in Anlehnung an den wissenschaftlichen "Gestaltbegriff», der hier mit dem «Formbegriff » synonym gesetzt wird - auf einem ästhetischen und ontologischen "Mehrwert », der einer Gestalt, einer Form zukomme. "Er bedeutet », sagt Musil über den Begriff der "Gestalt ", " daß aus dem Neben- oder Nacheinander sinnlich gegebener Elemente etwas entstehen kann, das sich nicht durch sie ausdrücken und ausmessen läßt ». (8 : 1218) Im Unterschied zur Formelbildung in unserem Alltag, die der ökonomischeren Bewältigung unserer Alltagsaufgaben dient, produziert die literarisch-ästhetische Form eine «neue Qualität»: "so besteht, als eines der einfachsten Beispiele, [...] eine Melodie aus ihren Tönen, aber in deren einmaligem Stand zueinander, der eben die Gestalt ausmacht und eine Ausdruck hat, der sich aus den Ausdrucksmöglichkeiten der Bestandteile nicht erklären läßt » $(8: 1218)$. In der « Form » des Kunstwerks liege seine « Individualität »begründet, sein « So und nicht wieder » $(8: 1218)$, seine rational nicht zu begründende Überzeugungskraft, aber auch seine « Ganzheit ». Mag die " Ganzheit » des literarischen Kunstwerks eine andere sein als die Ganzheit additiv zustandegekommener Summen, mag sie sich durch ihre spirituelle Überlegenheit von anderen Ganzheiten unterscheiden, was dennoch überrascht, ist dieser abrupte Rekurs des radikalen Identitäts- und Ganzheitsskeptikers Musil auf eben jene Vorstellungen von " Ganzheit ", « Gestalt » und « Form », gegen die er in seinen Texten anschreibt, die er in seinen Figuren ebenso wie in seinen essayistischen Partien erbarmungslos auflaufen lässt und auf die er in der Sorge um die Rechtfertigung einer zusehends von Marginalisierung betroffenen Literatur nicht verzichten mag. Die Literatur ist - so ließe sich folgern - die Literatur einer «besonderen Form ». Sie mag alle Gewissheiten zerstören, aber ihre eigene nicht; sie mag gegen alle Formen anschreiben und muss doch ihre eigene bewahren; sie mag ihre eigene Unwahrscheinlichkeit erkennen und muss dennoch auf ihrem Sein und Werden, auf ihrem Form-Sein und Form-Werden beharren. Die «Form» des Kunstwerks ist jener Code, der Kunstkommunikation 
reguliert, der letztlich aber auch die Plausibilität und Akzeptanz eines literarischen Textes garantiert.

\section{8.}

In unserem Bemühen, den Weg Musil'schen Schreibens und Denkens von seiner Prägung durch die krisenhaften Erfahrungen der Moderne hin zu einer Neudefinition literarischen Schreibens in der Moderne nachzuvollziehen, sind wir nun an unserem Ende angelangt. Wir enden - was Musil gefallen würde - mit einem Paradoxon; der Feststellung nämlich, dass auch das radikalste, illusionsloseste und gängige Zuschreibungen aufs Massivste verwerfende literarische Schreiben nicht darum herumkommt, für sich selbst in Anspruch zu nehmen, was es auf allen anderen Gebieten misstrauisch beäugt : die Behauptung einer formalen Identität. Mag in Musils Form-Konzept das Wissen um die Fragilität des Zustandekommens der Form, der Widersprüchlichkeit und jederzeit möglichen Aufkündigung formaler Ganzheit integriert sein, die Faszination der Form scheint unbestritten. In ihr vergewissert sich das verunsicherte schreibende Ich in der Moderne seiner unverwechselbaren Eigenheit wie seiner sozialen Notwendigkeit. Die literarische Form ist die literarische Form, und weil sie diese ist, vermag sie sich als spezifischen und unverzichtbaren Beitrag zu sozialer Kommunikation zu behaupten. Literatur kann noch so sehr ihre Aufgabe darin sehen, gegen falsche Identitätszuschreibungen und verlogene Identitätskonstruktionen anzuschreiben, wirksam wird sie nur, wenn sie selbst als «Form» unterscheidbar bleibt.

Der Faszination des Musil'schen Textuniversums tut dieser Befund keinen Abbruch. Er unterstreicht einmal mehr die labyrinthartige Verwobenheit unseres Denkens und Schreibens, das «neu » ist, wo es das Alte fortschreibt, und hausbacken, wo es sich " progressiv » wähnt. Was bleibt, ist vielleicht doch nur jenes Pathos der Genauigkeit, von dem Musil schreibt und an das wir uns weiter halten sollten.

\section{NOTES}

1. Robert Musil : «Profil eines Programms », in : R.M. : Gesammelte Werke in neun Bänden, Bd 8, hg. von Adolf Frisé. Reinbek bei Hamburg: Rowohlt 1981, S. 1315-1319, 1317. (Die Werke Robert Musils werden in der Folge nach der neunbändigen Werkausgabe mit Bandzahl und einfacher Seitenzahl zitiert.)

2. Schließlich sah sich der Ingenieur Musil ebenso wie später der Philosoph und Wahrnehmungstheoretiker Musil durchaus ernst zu nehmenden Angeboten aus Wirtschaft und Wissenschaft gegenüber.

3. Vgl. dazu ausführlicher : Alice Bolterauer : Rahmen und Riss. Robert Musil und die Moderne. Wien : Edition Praesens 2000.

4. Wilfried Berghahn : Robert Musil. Reinbek bei Hamburg: Rowohlt 1986, S. 13. Weiter heißt es dort: «Aus jeder sogenannten Tatsache lugt ein Stück Unwirklichkeit oder Noch-nichtWirklichkeit hervor. » 
5. Jean-François Lyotard: «Beantwortung der Frage: Was ist postmodern?», in: Wolfgang Welsch (Hg.): Wege aus der Moderne. Schlüsseltexte der Postmoderne-Diskussion. Berlin: Akademie Verlag 1994, S. 193-203.

6. «Es wäre wichtig, zu wissen, warum man sich bei einer roten Nase ganz ungenau damit begnügt, sie sei rot, und nie danach fragt, welches besondere Rot sie habe, obgleich sich das durch die Wellenlänge auf Mikromillimeter genau ausdrücken ließe» (1:9), heißt es gleich zu Beginn des Romans.

7. In seinem Aufsatz «Die Kunst als Verfahren » (1916) beschreibt Viktor Sklovskij das künstlerische Verfahren als «verfremdendes» Verfahren. Darin heißt es : «Das Verfahren der Verfremdung bei L. Tolstoj besteht darin, daß er einen Gegenstand nicht mit seinem Namen nennt, sondern ihn so beschreibt, als werde er zum ersten Mal gesehen, und einen Vorfall, als ob er sich zum ersten Mal ereigne [...]." Viktor Sklovskij: "Die Kunst als Verfahren", in: J. Striedter (Hg.) : Russischer Formalismus. München 1981, S. 4-35, 17.

8. Die Mathematik sei "Tapferkeitsluxus der reinen Ratio", heißt es in einer berühmten Formulierung Musils ( $8: 1006)$.

9. Hugo von Hofmannsthal : « Ein Brief », in : H.v.H. : Gesammelte Werke in zehn Einzelbänden, Bd 7, hg. von Bernd Schoeller und Rudolf Hirsch. Frankfurt/Main : Fischer 1979, S. 461-472, 465.

10. So wird dem Erstlingsroman «Die Verwirrungen des Zögling Törleß» ein ausführliches Maeterlinck-Zitat aus dessen « Der Schatz der Armen » vorangestellt.

11. Robert Musil : Tagebücher, Bd 1, hg. von Adolf Frisé. Reinbek bei Hamburg : Rowohlt 1983, S. 2. 12. Anlässlich einer Neuausgabe des Novellenbandes "Vereinigungen » schreibt Musil in sein Tagebuch: "Man sollte zwischen Glasplatten ein paar Seiten davon ausbreiten und sie von Zeit zu Zeit wechseln. Dann würde man sehen, was es ist. » Musil, Tagebücher, a.a.O., S. 347.

13. So wie Zeit und Ort ( $«$ Es soll also auf den Namen der Stadt kein besonderer Wert gelegt werden » (1: 10), heißt es), so werden auch die Personen nur vorgestellt, um sofort wieder zurückgenommen zu werden : « Angenommen, sie würden Arnheim und Ermelinda Tuzzi heißen, was aber nicht stimmt, denn Frau Tuzzi befand sich im August in Begleitung ihres Gatten in Bad Aussee und Dr. Arnheim noch in Konstantinopel, so steht man vor dem Rätsel, wer sie seien. » $(1: 10)$

14. Musil, Tagebücher, a.a.O., S. 934.

15. Zur Selbstreflexivität als Kennzeichen moderner Literatur im größeren Kontext der Wiener Moderne siehe auch: Alice Bolterauer: Selbstvorstellung. Die literarische Selbstreflexion der Wiener Moderne. Freiburg i. Br. : Rombach 2003.

16. Es handelt sich dabei um das berühmte 62. Kapitel im «Mann ohne Eigenschaften », das den ebenso zynischen wie ironischen, das eigene Erzählen in Frage stellenden Titel trägt : « Auch die Erde, namentlich aber Ulrich, huldigt der Utopie des Essayismus » $(1: 247-257)$.

17. «Die Figuren mögen fassungslos sein, der Text selbst ist es nicht ", habe ich einmal geschrieben in : Alice Bolterauer : «Marionetten und Männer ohne Eigenschaften. Überlegungen zur Identitätsproblematik bei Robert Musil», in: Hildegard Kernmayer (Hg.) : Zerfall und Rekonstruktion. Identitäten und ihre Repräsentation in der Österreichischen Moderne. Wien : Passagen 1999, S. 245-264, 262.

18. Niklas Luhmann : Soziale Systeme. Frankfurt/Main : Suhrkamp 1996.

19. Weil die Entscheidung für oder gegen die Literatur im Lebenslauf Musils immer auch eine Frage für oder gegen die Wissenschaft, d.h. über die Frage nach der Überlegenheit ästhetischer oder wissenschaftlich-rationaler Erkenntnis hinaus auch eine Entscheidungsfrage für oder gegen eine « ordentliche » Karriere gewesen ist.

20. Und nicht zufälligerweise war es Alexius Meinong, prominenter Vertreter der Grazer Schule der Gestalttheorie, der Robert Musil als Assistenten nach Graz holen wollte. 


\section{RÉSUMÉS}

Als grundlegendes Anliegen des österreichischen Autors Robert Musil kann sein Versuch angesehen werden, gegen falsche Identitäten und Identitätszuschreibungen aufzutreten. In dem Artikel werden die Krise der Wahrnehmung, die Krise der Erkenntnis, die Krise der Sprache und die Krise des Erzählens genauer erläutert, die auch als Identitätskrisen verstanden werden können. Im Gegensatz zu diesem identitätskritischen Diskurs steht aber die Betonung der literarischen Form, auf die - als Form - auch unter dem Zeichen der Moderne offensichtlich nicht verzichtet werden kann.

L'intention fondamentale de l'auteur autrichien Robert Musil consiste dans l'essai d'attaquer et de détruire chaque notion d'identité. Dans l'article que voici on thématise la crise de la perception, la crise du savoir et du parler comme celle du récit. Toutes ces crises peuvent être comprises comme manifestations et résultats d'une crise d'identité. Malgré cette position sceptique vis-à-vis de la notion d'identité, Robert Musil réclame l'idée d'une forme identique pour la littérature elle-même.

\section{AUTEUR}

\section{ALICE BOLTERAUER}

Universität Graz 